\section{Библиографический список}

1. Харди Г., Литтлвуд Дж., Полиа Г. Неравенства. M. : Изд-во иностр. лит., 1948. 456 с. [Hardy G., Littlewood J., Polya G. Inequalities. Cambridge Cambridge Univ. Press, 1934. 328 p.]

2. Крейн С. Г., Петунин Ю. И., Семенов Е. М. Интерполяция линейных операторов. М. : Наука, 1978. 400 с. [Krein S. G., Petunin J. I., Semenov E. M. Interpolation of linear operators. Providence: Amer. Math. Soc., 1982. 375 p.]

3. Титимари E. Введение в теорию интегралов Фурье. М.; Л. : Гостехиздат, 1948. 480 с. [Titchmarsh E. Introduction to the theory of Fourier integrals. Oxford : Clarendon Press, 1948. 404 p.]

УДК 517.982.22, 517.982.252+256, 519.615, 519.853.3

\section{МЕТОД ПРОЕКЦИИ ГРАДИЕНТА ДЛЯ СИЛЬНО ВЫПУКЛОГО МНОЖЕСТВА}

\section{М. О. Голубев}

Московский сризико-технический институт (государственный университет), Долгопрудный

E-mail: maksimkane@mail.ru

В работе рассматривается стандартный метод проекции градиента в случае, когда множество является $R$-сильно выпуклым, а фрунция выпукла, диффреренцируема и имеет липшицев градиент. Доказано, что при некоторых естественных дополнительных условиях метод сходится со скоростью геометрической прогрессии.

Ключевые слова: гильбертово пространство, метод проекции градиента, метрическая проекция, $R$-сильно выпуклое множество.
4. Голубов Б. И. Об одной теореме Беллмана о коэффициентах Фурье // Мат. сб. 1994. Т. 185, № 11. С. 3140. [Golubov B. I. On a Bellman theorem on Fourier coefficients // Russian Academy of Sciences. Sbornik. Mathematics. 1995. Vol. 83, № 2. P. 321-330.]

5. Moricz F. The harmonic Cesaro and Copson operators on the spaces $L^{p}(\mathbb{R}), 1 \leq p \leq 2 / /$ Studia Math. 2002. Vol. 149, № 3. P. 267-279.

6. Зигмунд A. Тригонометрические ряды : в 2 т. Т. 1. M. : Мир, 1965. 616 с. [Zygmund A. Trigonometric series. Vol. 1. Cambridge : Cambridge Univ. Press, 1959. 320 p.]

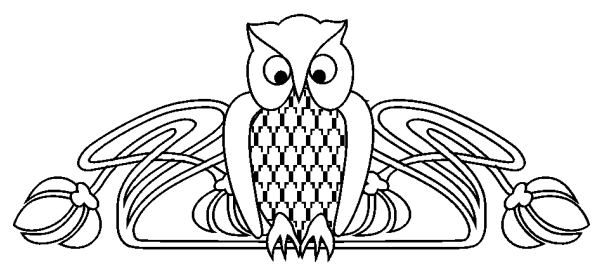

\title{
M. O. Golubev
}

In our work we will discuss standard gradient projection algorithm, where a set is strongly convex of radius $R$ and a function is convex, differentiable and its gradient satisfies Lipschitz condition. We proved that under some natural additional conditions algorithm converges with the rate of a geometric progression.

Key words: Hilbert space, gradient projection algorithm, metric projection, strongly convex set of radius $R$.

\section{ВВЕДЕНИЕ}

Пусть $\mathbb{H}$ - гильбертово пространство над вещественным полем скаляров, $(p, x)$ - скалярное произведение векторов $p, x \in \mathbb{H}$. Обозначим через $B_{R}(x)=\{y \in \mathbb{H}:\|y-x\| \leq R\}$ замкнутый шар радиуса $R \geq 0$ с центром в точке $x \in \mathbb{H}$. Расстояние от точки $x \in \mathbb{H}$ до множества $A \subset \mathbb{H}$ будем обозначать $\varrho(x, A) \doteq \inf \{\|x-a\|: a \in A\}$. Метрической проекцией точки $x \in \mathbb{H}$ на множество $A \subset \mathbb{H}$ называется множество $P_{A}(x)=\{a \in A:\|x-a\|=\varrho(x, A)\}$. Опорная функция ко множеству $A$ определяется следующей формулой: $s(p, A)=\sup _{x \in A}(p, x)$ для всех $p \in \mathbb{H}$. Нормальным конусом к выпуклому замкнутому множеству $A$ в точке $a \in A$ называется множество $N(A ; a)=\{p \in \mathbb{H}:(p, a) \geq s(p, A)\}$. Диаметром множества $A$ называется число $\operatorname{diam} A=\sup _{x, y \in A}\|x-y\|$. Границу множества $A$ обозначим через $\partial A$.

Определение $1[1$, определение $3.1 .1 ; 2,3]$. Непустое множество $A \subset \mathbb{H}$ называется $R$-сильно выпуклым, если оно может быть представлено в виде пересечения замкнутых шаров радиуса $R>0$, т. е. $A=\bigcap_{x \in X} B_{R}(x)$ для некоторого подмножества $X \subset \mathbb{H}$.

Рассмотрим задачу минимизации:

$$
f(x) \rightarrow \min , \quad x \in A \subset \mathbb{H} .
$$

В данной работе мы обсудим стандартный метод проекции градиента:

$$
x_{k+1}=P_{A}\left(x_{k}-\alpha_{k} f^{\prime}\left(x_{k}\right)\right), \quad x_{1} \in \partial A, \quad \alpha_{k}>0 .
$$

Метод проекции градиента детально изложен в работах [4-7]. Известные случаи сходимости метода проекции градиента со скоростью геометрической прогрессии имеют место для замкнутого и выпуклого множества $A$ и сильно выпуклой с константой $\theta>0$ функции $f$, градиент $f^{\prime}$ которой удовлетворяет 
условию Липшица с константой $M>0$, т. е. $\left\|f^{\prime}\left(x_{1}\right)-f^{\prime}\left(x_{2}\right)\right\| \leq M\left\|x_{1}-x_{2}\right\|$ для всех $x_{1}, x_{2} \in \mathbb{H}$. В работе [8] приведена следующая оценка скорости сходимости $\left\|x_{k+1}-x_{*}\right\| \leq q\left\|x_{k}-x_{*}\right\|$, где $x_{*}-$ единственное решение задачи (1) и $q=\sqrt{1-4 \theta \alpha+\alpha^{2} M^{2}}$, а коэффициенты $\alpha_{k}$ выбираются с учетом условия $\alpha_{k}=\alpha$, а $\alpha \in\left(0,4 \theta / M^{2}\right)$. Мы планируем отказаться от сильной выпуклости функции $f$, но потребуем сильной выпуклости множества $A$.

Теорема 1 была анонсирована в тезисах конференций $[9,10]$.

\section{1. ВСПОМОГАТЕЛЬНЫЕ РЕЗУЛЬТАТЫ}

Предложение 1 [1, теорема 4.1.3]. Замкнутое выпуклое множество $A \subset \mathbb{H}$ является $R$-сильно выпуклым множеством тогда и только тогда, когда оно представимо в виде

$$
A=\bigcap_{\|p\|=1} B_{R}\left(x_{p}-R p\right),
$$

где для любого вектора $p \in \mathbb{H},\|p\|=1$ точка $x_{p} \in A$ однозначно определена из равенства $\left(p, x_{p}\right)=s(p, A)$.

Предложение 2 [1, лемма 2.2, гл. 6]. Пусть множество $A \subset \mathbb{H}$ выпукло и замкнуто, функция $f: \mathbb{H} \rightarrow \mathbb{R}$ выпуклана $A$ и дифференцируема в точке $x_{*} \in A$.

Тогда $x_{*}$ является решением задачи (1) в том и только том случае, если

$$
x_{*}=P_{A}\left(x_{*}-\alpha f^{\prime}\left(x_{*}\right)\right)
$$

при произвольном $\alpha>0$.

Хорошо известно, что для выпуклого и замкнутого множества $A \subset \mathbb{H}$ множество $P_{A}(x)$ одноточечно, т. е. $P_{A}(x)=\{a(x)\}$, и для любых точек $x_{0}, x_{1} \in \mathbb{H}$ выполняется оценка $\left\|a_{0}-a_{1}\right\| \leq 1 \cdot\left\|x_{0}-x_{1}\right\|$, где $\left\{a_{i}\right\}=P_{A}\left(x_{i}\right), i \in\{0,1\}$.

Для выпуклого замкнутого множества $A \subset \mathbb{H}$ и вектора $p \in \mathbb{H}$ определим множество $A(p)=\{x \in A:(p, x)=s(p, A)\}$.

Предложение 3 [1, теорема 3.1.3]. Замкнутое выпуклое множество $A \subset \mathbb{H}$ является $R$-сильно выпуклым множеством тогда и только тогда, когда для любой пары единичных векторов $p, q \in \mathbb{H}$ и для точек $\{a(p)\}=A(p),\{a(q)\}=A(q)$ выполняется следующее неравенство:

$$
\|a(p)-a(q)\| \leq R\|p-q\|
$$

Теорема 1. Пусть множество $A \subset \mathbb{H}$ является $R$-сильно выпуклым множеством. Тогда для любых точек $x_{0}, x_{1} \in \mathbb{H} \backslash A$ выполнено неравенство

$$
\left\|a_{0}-a_{1}\right\| \leq \frac{R}{\sqrt{\left(R+\varrho_{0}\right)\left(R+\varrho_{1}\right)}} \cdot \sqrt{\left\|x_{0}-x_{1}\right\|^{2}-\left(\varrho_{0}-\varrho_{1}\right)^{2}},
$$

где $\left\{a_{i}\right\}=P_{A}\left(x_{i}\right), \varrho_{i}=\left\|x_{i}-a_{i}\right\|, i \in\{0,1\}$.

Доказательство. Из предложения 3 следует, что

$$
\left\|a_{0}-a_{1}\right\| \leq R\left\|\frac{x_{0}-a_{0}}{\varrho_{0}}-\frac{x_{1}-a_{1}}{\varrho_{1}}\right\| .
$$

После возведения данного неравенства в квадрат имеем:

$$
\begin{gathered}
\left\|a_{0}-a_{1}\right\|^{2} \leq R^{2}\left(2-\frac{2}{\varrho_{0} \varrho_{1}}\left(x_{0}-a_{0}, x_{1}-a_{1}\right)\right)= \\
=R^{2}\left(2+\frac{\left\|a_{0}-a_{1}\right\|^{2}+\left\|x_{0}-x_{1}\right\|^{2}-\left\|a_{0}-x_{1}\right\|^{2}-\left\|a_{1}-x_{0}\right\|^{2}}{\varrho_{0} \varrho_{1}}\right) .
\end{gathered}
$$

Из предложения 1 следует, что

$$
A \subset B_{R}\left(a_{0}-R \frac{x_{0}-a_{0}}{\varrho_{0}}\right) .
$$


Пусть $y=a_{0}-R \frac{x_{0}-a_{0}}{\varrho_{0}} .\left\|y-a_{1}\right\| \leq R$, так как $a_{1} \in B_{R}(y)$. Заметим, что $\angle x_{0} a_{0} a_{1}=\pi-\angle y a_{0} a_{1}$.

По теореме косинусов из треугольника $у a_{0} a_{1}$ следует

$$
\begin{gathered}
\cos \angle x_{0} a_{0} a_{1}=-\cos \angle y a_{0} a_{1}=-\frac{\left\|y-a_{0}\right\|^{2}+\left\|a_{0}-a_{1}\right\|^{2}-\left\|y-a_{1}\right\|^{2}}{2\left\|y-a_{0}\right\|\left\|a_{0}-a_{1}\right\|}= \\
=-\frac{R^{2}+\left\|a_{0}-a_{1}\right\|^{2}-\left\|y-a_{1}\right\|^{2}}{2 R\left\|a_{0}-a_{1}\right\|} \leq-\frac{\left\|a_{0}-a_{1}\right\|}{2 R} .
\end{gathered}
$$

По теореме косинусов из треугольника $x_{0} a_{0} a_{1}$ имеем:

$$
\begin{gathered}
\left\|a_{1}-x_{0}\right\|^{2}=\left\|a_{0}-a_{1}\right\|^{2}+\left\|a_{0}-x_{0}\right\|^{2}-2\left\|a_{0}-a_{1}\right\|\left\|a_{0}-x_{0}\right\| \cos \angle x_{0} a_{0} a_{1} \geq \\
\geq\left\|a_{0}-a_{1}\right\|^{2}+\varrho_{0}^{2}+\frac{\left\|a_{0}-a_{1}\right\|^{2} \varrho_{0}}{R} .
\end{gathered}
$$

Аналогичным образом получаем неравенство

$$
\left\|a_{0}-x_{1}\right\|^{2} \geq\left\|a_{0}-a_{1}\right\|^{2}+\varrho_{1}^{2}+\frac{\left\|a_{0}-a_{1}\right\|^{2} \varrho_{1}}{R} .
$$

Из формулы (5) имеем:

$$
\left\|a_{0}-a_{1}\right\|^{2} \leq R^{2}\left(2+\frac{\left\|x_{0}-x_{1}\right\|^{2}-\varrho_{1}^{2}-\frac{\left\|a_{0}-a_{1}\right\|^{2}\left(\varrho_{1}+\varrho_{0}\right)}{R}-\left\|a_{0}-a_{1}\right\|^{2}-\varrho_{0}^{2}}{\varrho_{0} \varrho_{1}}\right),
$$

поэтому

$$
\left\|a_{0}-a_{1}\right\|^{2}\left(R^{2}+R\left(\varrho_{0}+\varrho_{1}\right)+\varrho_{0} \varrho_{1}\right) \leq R^{2}\left(\left\|x_{0}-x_{1}\right\|^{2}-\left(\varrho_{0}-\varrho_{1}\right)^{2}\right) .
$$

После преобразований получаем следующую оценку:

$$
\left\|a_{0}-a_{1}\right\| \leq \frac{R}{\sqrt{\left(R+\varrho_{0}\right)\left(R+\varrho_{1}\right)}} \cdot \sqrt{\left\|x_{0}-x_{1}\right\|^{2}-\left(\varrho_{0}-\varrho_{1}\right)^{2}} .
$$

Замечание 1. В работе [8] аналогичная оценка была получена для выпуклых множеств с $C^{2}$ гладкой границей.

Замечание 2. Заметим, что если $x_{0} \in A$ (т. е. $\varrho_{0}=0$ ), то формула (4) остается верной. В этом случае $a_{0}=P_{A}\left(x_{0}\right)=x_{0}$. В силу предложения 1

$$
x_{0} \in A \subset B_{R}\left(a_{1}-R \frac{x_{1}-a_{1}}{\varrho_{1}}\right) .
$$

По аналогии с доказательством теоремы 1 имеем $\cos \angle x_{0} a_{1} x_{1} \leq-\frac{\left\|a_{0}-a_{1}\right\|}{2 R}$. По теореме косинусов из треугольника $x_{0} a_{1} a_{0}$ получаем оценку

$$
\left\|a_{0}-x_{1}\right\|^{2}=\left\|a_{1}-x_{1}\right\|^{2}+\left\|a_{0}-a_{1}\right\|^{2}-2\left\|a_{1}-x_{1}\right\|\left\|a_{0}-a_{1}\right\| \cos \angle x_{0} a_{1} x_{1} .
$$

В силу того что $a_{0}=x_{0}$, имеем:

$$
\begin{gathered}
\left\|x_{0}-x_{1}\right\|^{2}=\left\|a_{1}-x_{1}\right\|^{2}+\left\|a_{0}-a_{1}\right\|^{2}-2\left\|a_{1}-x_{1}\right\|\left\|a_{0}-a_{1}\right\| \cos \angle x_{0} a_{1} x_{1} \geq \\
\geq \varrho_{1}^{2}+\left\|a_{0}-a_{1}\right\|^{2}+\frac{\left\|a_{0}-a_{1}\right\|^{2} \varrho_{1}}{R} .
\end{gathered}
$$

Из формулы (6) следует, что

$$
\left\|a_{0}-a_{1}\right\| \leq \sqrt{\frac{R}{R+\varrho_{1}}} \cdot \sqrt{\left\|x_{0}-x_{1}\right\|^{2}-\varrho_{1}^{2}} .
$$

Последнее эквивалентно формуле (4) в случае, когда $x_{0}=a_{0}$ и $\varrho_{0}=0$.

Предложение 4. [1, лемма 1.19.5]. Пусть функция $f: \mathbb{H} \rightarrow \mathbb{R}$ выпукла и дифференцируема по Гато на $\mathbb{H}$. Тогда условие Липиица для градиента $f^{\prime}$

$$
\left\|f^{\prime}\left(x_{1}\right)-f^{\prime}\left(x_{2}\right)\right\| \leq M\left\|x_{1}-x_{2}\right\|, \quad \forall x_{1}, x_{2} \in \mathbb{H},
$$

эквивалентно условию

$$
\left(f^{\prime}\left(x_{1}\right)-f^{\prime}\left(x_{2}\right), x_{1}-x_{2}\right) \geq \frac{1}{M}\left\|f^{\prime}\left(x_{1}\right)-f^{\prime}\left(x_{2}\right)\right\|^{2}, \quad \forall x_{1}, x_{2} \in \mathbb{H} .
$$




\section{2. ОСНОВНОЙ РЕЗУЛЬТАТ}

Рассмотрим задачу (1). Последовательность $x_{k}$ генерируется по правилу (2).

Предположим, что

1) непустое множество $A \subset \mathbb{H}$ является $R$-сильно выпуклым (т. е. $A=\bigcap_{x \in X} B_{R}(x) \neq \emptyset$ );

2 ) функция $f: \mathbb{H} \rightarrow \mathbb{R}$ является выпуклой, дифференцируемой, и градиент $f^{\prime}(x)$ удовлетворяет условию Липшица с константой $M>0$ : для любой пары точек $x_{1}, x_{2} \in \mathbb{H}$ выполнено

$$
\left\|f^{\prime}\left(x_{1}\right)-f^{\prime}\left(x_{2}\right)\right\| \leq M\left\|x_{1}-x_{2}\right\| ;
$$

3) для всех $k \in \mathbb{N}$ существует вектор $n\left(x_{k}\right) \in N\left(A, x_{k}\right)$, такой что выполняется неравенство $\left(n\left(x_{k}\right), f^{\prime}\left(x_{k}\right)\right) \leq 0$ (т. е. $x_{k}-\alpha_{k} f^{\prime}\left(x_{k}\right) \notin A$ для любого $\left.\alpha_{k}>0\right)$;

4) решение задачи (1) $x_{*} \in \partial A$ единственно;

5) $t=\min _{x \in \partial A}\left\|f^{\prime}(x)\right\|>0$.

Заметим, что условие 2) для выпуклой функции эквивалентно условию (7).

В случае, когда условие 3) не выполняется, мы имеем дело с безусловной минимизацией и следует использовать один из стандартных алгоритмов поиска безусловного минимума (см. например [7, теорема 1.2 , теорема 2.1 , гл. 5]).

Теорема 2. 1. Пусть выполнены условия 1)-5). Пусть $\alpha_{k}=\alpha \in(0,2 / M]$. Тогда последовательность $x_{k}$, генерируемая по правилу (2), сходится к решению задачи (1) со скоростью геометрической прогрессии: $\left\|x_{k+1}-x_{*}\right\| \leq q\left\|x_{k}-x_{*}\right\|$, где $q=\frac{R}{\sqrt[4]{\left(R^{2}+\alpha^{2} t^{2}\right)(R+\alpha t)^{2}}}$;

2. Пусть выполнены условия 1)-4). Пусть $\alpha_{k}=\alpha \in(0,2 / M]$. Тогда последовательность $x_{k}$, генерируемая по правилу (2), сходится к решению задачи (1) со скоростью: $\left\|x_{k+1}-x_{*}\right\| \leq q_{k}\left\|x_{k}-x_{*}\right\|$, где $q_{k}=\sqrt[4]{\frac{R^{2}}{R^{2}+\alpha^{2}\left\|f^{\prime}\left(x_{k}\right)\right\|^{2}}}$.

Доказательство. Воспользуемся предложением 1. Шар $B_{R}\left(x_{k}-R n\left(x_{k}\right)\right)$ содержит множество $A$, где единичный вектор $n\left(x_{k}\right)$ из условия 3$)$. Вектор $f^{\prime}\left(x_{k}\right)$ по условию составляет тупой угол с вектором $n\left(x_{k}\right)$. Пусть $y_{k} \doteq x_{k}-\alpha f^{\prime}\left(x_{k}\right), z_{k} \doteq x_{k}-R n\left(x_{k}\right)$. Пусть $\varrho_{x_{k}}=\varrho\left(x_{k}-\alpha f^{\prime}\left(x_{k}\right), A\right)$, $\varrho_{x_{*}}=\varrho\left(x_{*}-\alpha f^{\prime}\left(x_{*}\right), A\right)$. Далее, применяя теорему косинусов для треугольника $x_{k} y_{k} z_{k}$, получаем: $\varrho_{x_{k}} \geq \sqrt{R^{2}+\alpha^{2}\left\|f^{\prime}\left(x_{k}\right)\right\|^{2}}-R$. Из формулы (3) следует, что $\varrho_{x_{*}}=\alpha\left\|f^{\prime}\left(x_{*}\right)\right\|$.

Следуя формуле (4), определим для точек $x_{k}, x_{*} \in \partial A$ и чисел $R>0, \alpha \in(0,2 / M]$ число

$$
L_{k}=L\left(x_{k}, x_{*}, R, \alpha\right)=\frac{R}{\sqrt[4]{\left(R^{2}+\alpha^{2}\left\|f^{\prime}\left(x_{k}\right)\right\|^{2}\right)} \sqrt{\left(R+\alpha\left\|f^{\prime}\left(x_{*}\right)\right\|\right)^{2}}} .
$$

Воспользовавшись неравенством (4), имеем

$$
\begin{aligned}
\left\|x_{k+1}-x_{*}\right\|^{2} & =\left\|P_{A}\left(x_{k}-\alpha f^{\prime}\left(x_{k}\right)\right)-P_{A}\left(x_{*}-\alpha f^{\prime}\left(x_{*}\right)\right)\right\|^{2} \leq L_{k}^{2}\left\|\left(x_{k}-x_{*}\right)-\left(\alpha f^{\prime}\left(x_{k}\right)-\alpha f^{\prime}\left(x_{*}\right)\right)\right\|^{2}= \\
& =L_{k}^{2}\left(\left\|x_{k}-x_{*}\right\|^{2}-2 \alpha\left(x_{k}-x_{*}, f^{\prime}\left(x_{k}\right)-f^{\prime}\left(x_{*}\right)\right)+\alpha^{2}\left\|f^{\prime}\left(x_{k}\right)-f^{\prime}\left(x_{*}\right)\right\|^{2}\right) .
\end{aligned}
$$

Из (7) следует, что

$$
\left\|x_{k+1}-x_{*}\right\|^{2} \leq L_{k}^{2}\left(\left\|x_{k}-x_{*}\right\|^{2}+\left(\alpha^{2}-2 \frac{\alpha}{M}\right)\left\|f^{\prime}\left(x_{k}\right)-f^{\prime}\left(x_{*}\right)\right\|^{2}\right) .
$$

Так как по условию теоремы $\alpha \in(0,2 / M]$, то $\left\|x_{k+1}-x_{*}\right\|^{2} \leq L_{k}^{2}\left\|x_{k}-x_{*}\right\|^{2}$.

Отсюда получаем оценку

$$
\left\|x_{k+1}-x_{*}\right\| \leq \frac{R}{\sqrt[4]{\left(R^{2}+\alpha^{2}\left\|f^{\prime}\left(x_{k}\right)\right\|^{2}\right)} \sqrt{\left(R+\alpha\left\|f^{\prime}\left(x_{*}\right)\right\|\right)^{2}}}\left\|x_{k}-x_{*}\right\| .
$$

В случае 1) $q=\frac{R}{\sqrt[4]{\left(R^{2}+\alpha^{2} t^{2}\right)} \sqrt{(R+\alpha t)^{2}}}$. В случае 2) $q_{k}=\sqrt[4]{\frac{R^{2}}{R^{2}+\alpha^{2}\left\|f^{\prime}\left(x_{k}\right)\right\|^{2}}}$.

Теорема 3. Пусть выполнены условия 1)-2). Пусть $R M / t<1$, где $t=\min _{x \in \partial A}\left\|f^{\prime}(x)\right\|>0$.

Последовательность $x_{k}$ генерируется по правилу (2) с $\alpha_{k}=\alpha>0$ для всех $k$. 
Tогда:

1) при выборе $\alpha \in(2 R / t, 2 / M]$ последовательность $x_{k}$ сходится $\kappa$ решению задачи (1) со скоростью геометрической прогрессии: $\left\|x_{k+1}-x_{*}\right\| \leq q(\alpha)\left\|x_{k}-x_{*}\right\|$, где $q(\alpha)=\frac{R}{\alpha t-R}$;

2) при выборе $\alpha>2 / M$ последовательность $x_{k}$ сходится $\kappa$ решению задачи (1) со скоростью геометрической прогрессии: $\left\|x_{k+1}-x_{*}\right\| \leq q(\alpha)\left\|x_{k}-x_{*}\right\|$, где $q(\alpha)=\frac{R(\alpha M-1)}{\alpha t-R}$. Более того, $q(\alpha) \underset{\alpha \rightarrow \infty}{\longrightarrow} R M / t<1$.

Доказательство. Заметим, что с учетом выбора параметра $\alpha$ в случае 1) $\alpha>2 R / t$. В случае 2 ) из неравенства $R M / t<1$ с учетом выбора параметра $\alpha$ выполняется неравенство $\alpha>2 R / t$. Таким образом, в обоих случаях верно:

$$
\alpha>\frac{2 R}{t}
$$

Рассмотрим произвольную точку $x \in \partial A$. Воспользуемся предложением 1. Существует вектор $n \in \mathbb{H},\|n\|=1, s(n, A)=(n, x)$ такой, что выполнено включение $A \subset B_{R}(x-R n)$.

Пусть $z \doteq x-R n, y \doteq x-\alpha f^{\prime}(x)$. Из неравенства треугольника следует, что $\|y-z\|+\|z-x\| \geq$ $\geq\|y-x\|$, отсюда $\|y-z\| \geq \alpha\left\|f^{\prime}(x)\right\|-R>\frac{2 R}{t} t-R=R$.

Таким образом, для любого $x \in \partial A$ выполнено включение $x-\alpha f^{\prime}(x) \notin B_{R}(x-R n)$, отсюда $x-\alpha f^{\prime}(x) \notin A$.

Оценим для $x \in \partial A$ число $\varrho_{x}=\varrho\left(x-\alpha f^{\prime}(x), A\right)$ :

$$
\varrho_{x} \geq \varrho\left(x-\alpha f^{\prime}(x), B_{R}(x-R n)\right)=\|y-z\|-R \geq \alpha\left\|f^{\prime}(x)\right\|-2 R .
$$

Таким образом, с учетом неравенства (8) $\varrho_{x} \geq \alpha t-2 R>0$.

Пусть $x, y \in \partial A$. Введем отображение $B$ вида $B x=P_{A}\left(x-\alpha f^{\prime}(x)\right)$. Для точек $x, y \in \partial A$ и чисел $R>0, \alpha$ определим величину $L_{x, y}=L(x, y, R, \alpha)=\frac{R}{\sqrt{\left(R+\varrho_{x}\right)\left(R+\varrho_{y}\right)}}$, где $\varrho_{x}=\varrho\left(x-\alpha f^{\prime}(x), A\right)$, $\varrho_{y}=\varrho\left(x-\alpha f^{\prime}(x), A\right)$. Величина $\varrho_{y}$ оценивается аналогично $\varrho_{x}$, следовательно, $\varrho_{y} \geq \alpha t-2 R$. Таким образом, $L_{x, y} \leq \frac{R}{\alpha t-R}$, учитывая неравенство $\alpha>\frac{2 R}{t}$ имеем: $L_{x, y}<1$

$$
\begin{gathered}
\|B x-B y\|^{2}=\left\|P_{A}\left(x-\alpha f^{\prime}(x)\right)-P_{A}\left(y-\alpha f^{\prime}(y)\right)\right\|^{2} \leq \\
\leq L_{x, y}^{2}\left\|x-\alpha f^{\prime}(x)-\left(y-\alpha f^{\prime}(y)\right)\right\|^{2} \leq L_{x, y}^{2}\left\|(x-y)-\alpha\left(f^{\prime}(x)-f^{\prime}(y)\right)\right\|^{2}= \\
=L_{x, y}^{2}\left(\|x-y\|^{2}+\alpha^{2}\left\|f^{\prime}(x)-f^{\prime}(y)\right\|^{2}-2 \alpha\left(x-y, f^{\prime}(x)-f^{\prime}(y)\right)\right), \\
\|B x-B y\|^{2} \leq L_{x, y}^{2}\left(\|x-y\|^{2}+\alpha^{2}\left\|f^{\prime}(x)-f^{\prime}(y)\right\|^{2}-\frac{2 \alpha}{M}\left\|f^{\prime}(x)-f^{\prime}(y)\right\|^{2}\right) .
\end{gathered}
$$

В случае 1$)$ выполнено $\alpha \in(2 R / t, 2 / M)$. Из неравенства (9) получаем оценку

$$
\|B x-B y\| \leq L_{x, y}\|x-y\| \leq \frac{R}{\alpha t-R}\|x-y\|,
$$

причем $\frac{R}{\alpha t-R}<1$ в силу выбора числа $\alpha$.

В случае 2) выполнено неравенство $\alpha>2 / M$. Из неравенств (9) и (7) получаем оценку

$$
\|B x-B y\| \leq L_{x, y}(\alpha M-1)\|x-y\| \leq \frac{R(\alpha M-1)}{\alpha t-R}\|x-y\| .
$$

С учетом условий $R M / t<1$ и $\alpha>\frac{2}{M}$ следует $\frac{R(\alpha M-1)}{\alpha t-R}<1$.

Таким образом отображение $B$ сжимающее. Множество $A$ является полным метрическим пространством. В силу принципа сжимающих отображений для процесса (2) имеем $x_{n} \rightarrow x_{*}$ при $n \rightarrow \infty$. Точка $x_{*}$ является неподвижной точкой отображения $B$. Из предложения 2 следует, что точка $x_{*}$ является решением задачи (1).

Из неравенств (10) и (11) следует оценка:

$$
\left\|x_{k+1}-x_{*}\right\|=\left\|B\left(x_{k}\right)-B\left(x_{*}\right)\right\| \leq q(\alpha)\left\|x_{k}-x_{*}\right\| .
$$


В случае 1) $q(\alpha)=\frac{R}{\alpha t-R}$. В случае 2) $q(\alpha)=\frac{R(\alpha M-1)}{\alpha t-R}$. Причем легко видеть, что
$\frac{R(\alpha M-1)}{\alpha t-R} \underset{\alpha \rightarrow \infty}{\longrightarrow} \frac{R M}{t}<1$.

Работа выполнена при финансовой поддержке РФФИ (проект 10-01-00139-а).

\section{Библиографрический список}

1. Половинкин E. С., Балашов М. В. Элементы выпуклого и сильно выпуклого анализа. М. : Физматлит, 2007. 440 c. [Polovinkin E. S. Balashov M. V. Elements of convex and strongly convex analysis. Moscow : Fizmatlit, 2007. 440 p.]

2. Поляк Б. Т. Теоремы существования и сходимость минимизирующих последовательностей в задачах на экстремум при наличии ограничений // Докл. АН CCCP. 1966. T. 166, №2. C. 287-290. [Polyak B. T. Existence theorems and convergence of minimizing sequences in extremal problems with restrictions // Soviet Math. Dokl. 1966. Vol. 7. P. 72-75.]

3. Поляк Б. Т., Левинтин Е. С. Сходимость минимизирующих последовательностей в задачах на условный экстремум // Докл. АН СССР. 1966. Т. 168, №5. C. 997-1000. [Polyak B. T., Levintin E. S. Convergence of minimizing sequences in conditional extremum problems // Soviet Math. Dokl. 1966. Vol. 7. P. 764-767.]

4. Васильев Ф. П. Численные методы решения экстремальных задач. M. : Наука, 1980. 520 с. [Vasilyev F. P. Numerical methods for solving extremal problems. Moscow : Nauka, 1980. 520 p.]

5. Нестеров Ю. Е. Введение в выпуклую оптимизацию. М. : МЦНМО, 2010. 279 с. [Nesterov Yu. E. Introduction to convex optimization. M. : MCCME, 2010. 279 p.]

6. Поляк Б. Т. Введение в оптимизацию. М. : Наука, 1983. 384 c. [Polyak B. T. Introduction to optimization. Moscow : Nauka, 1983. 384 p.]

7. Сухарев А. Г., Тимохов А. В., Федоров В. В. Курс методов оптимизации. М. : Физматлит, 2005. 368 с. [Sukharev A. G., Timokhov A. V., Fedorov V. V. Course of optimization methods. Moscow : Fizmatlit, 2005. 368 p.]

8. Abatzoglou T. J. The Lipschitz continuity of the metric projection // J. of Approx. Theory. 1979. Vol. 26. P. 212218.

9. Балашов М. В., Голубев М. О. Об условии Липшица для метрической проекции в гильбертовом пространстве // Тр. 54-й науч. конф. МФТИ. М. : МФТИ, 2011. T. 1. C. 34. [Balashov M. V. Golubev M. O. Lipschitz condition for the metric projection in a Hilbert space // Proc. of the 54th Conf. of MIPT. Moscow : MIPT, 2011. Vol. 1. P. 34.]

10. Голубев М. О. Метрическая проекция в гильбертовом пространстве и сильная выпуклость // Современные проблемы теории функций и их приложения : материалы 16-й Сарат. зимней шк. Саратов : Научная книга, 2012. C. 55-56. [Golubev M. O. Metric projection in a Hilbert space and strong convexity // Modern problems of function theory and their applications : Proc. of the 16th Saratov Winter School. Saratov, 2012. P. 55-56.]

УДК 517.51

\section{АБСОЛЮТНАЯ СХОДИМОСТЬ РЯДОВ, СВЯЗАННЫХ С РЯДАМИ ФУРЬЕ-ВИЛЕНКИНА}

\section{Н. В. Егошина}

Саратовский государственный университет E-mail: saviour92@mail.ru

Две теоремы О. П. Гойяла, касающиеся абсолютной сходимости некоторых тригонометрических рядов, распространяются на случай систем Виленкина и $L^{p}$-модулей непрерывности.

Ключевые слова: мультипликативные системы, положительные коэсрфициенты Фурье-Виленкина, абсолютная сходимость.

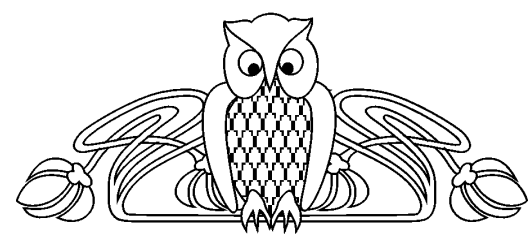

Absolute Convergence of Some Series, Connected with the Fourier-Vilenkin Series

\section{N. V. Egoshina}

Two theorems of O. P. Goyal concerning absolute convergence of some trigonometric series are extended to the case of Vilenkin systems and $L^{p}$-modulus of continuity.

Key words: positive Fourier-Vilenkin coefficients, absolute convergence.

\section{ВВЕДЕНИЕ}

Пусть $\mathbf{P}=\left\{p_{j}\right\}_{j=1}^{\infty}-$ последовательность натуральных чисел такая, что $2 \leq p_{j} \leq N$ при всех $j \in \mathbb{N}$ и $\mathbb{Z}_{j}=\left\{0,1, \ldots, p_{j}-1\right\}$. По определению полагаем $m_{0}=1, m_{n}=p_{1} \ldots p_{n}$ при $n \in \mathbb{N}$. Тогда каждое число $x \in[0,1)$ имеет разложение

$$
x=\sum_{j=1}^{\infty} x_{j} m_{j}^{-1}, \quad x_{j} \in \mathbb{Z}_{j}
$$

